\title{
Assessment of frequency and severity of hypomagnesemia in patients with metastatic colorectal cancer treated with cetuximab, with a review of the literature
}

\author{
JOANNA STREB ${ }^{1}$, MIROSLAWA PÜSKÜLLÜOĞLU ${ }^{1}$, IZABELA GLANOWSKA ${ }^{1}$, SEBASTIAN OCHENDUSZKO ${ }^{1}$, \\ KAMIL KONOPKA ${ }^{1}$, RADOSLAW ŁUPKOWSKI ${ }^{1}$, ANNA MICHALOWSKA-KACZMARCZYK ${ }^{1}$, \\ JUSTYNA BOCHENEK-CIBOR ${ }^{2}$, MARCIN MAJKA ${ }^{3}$ and KRZYSZTOF KRZEMIENIECKI ${ }^{1}$ \\ ${ }^{1}$ Department of Clinical Oncology, University Hospital, Jagiellonian University Medical College, Kraków 31-008; \\ ${ }^{2}$ Department of Radiation Therapy, St. Luke's Hospital, Tarnów 33-100; ${ }^{3}$ Department of Transplantation, \\ Polish-American Institute of Pediatrics, Jagiellonian University Medical College, Kraków 31-008, Poland
}

Received September 23, 2014; Accepted June 16, 2015

DOI: $10.3892 / \mathrm{ol} .2015 .3800$

\begin{abstract}
Currently, there are a few systemic treatment options for patients with metastatic colorectal cancer (mCRC). Targeted therapy used in this setting includes the use of monoclonal antibodies, such as cetuximab or panitumumab, directed against epidermal growth factor receptor. The aim of the present study was to estimate the frequency and severity of hypomagnesemia among patients with mCRC treated with cetuximab. The data from the Department of Clinical Oncology, University Hospital of Krakow (Krakow, Poland), concerning 52 patients treated between 2009 and 2013 were collected. Of these, 27 patients fulfilled the inclusion criteria to enter this retrospective study. The National Cancer Institute Common Terminology Criteria for Adverse Events version 4.0 were used to grade the level of hypomagnesemia. In total, $29.6 \%$ of all patients experienced hypomagnesemia during treatment, and the majority of cases were grade $1(22.2 \%)$. There was no statistically significant correlation between magnesium $(\mathrm{Mg})$ level and patient age, duration of treatment, localization of primary tumor or metastases, and the number of metastases. However, there was an upward trend in a logistic regression model showing that the risk of developing hypomagnesemia increases with age. Hypomagnesemia is a frequent problem among mCRC patients receiving cetuximab. It is essential to introduce guidelines regarding the monitoring of the $\mathrm{Mg}$ level and its supplementation in this group of patients.
\end{abstract}

Correspondence to: Dr Mirosława Püsküllüoğlu, Department of Clinical Oncology, University Hospital, Jagiellonian University Medical College, 10 Śniadeckich Street, Kraków 31-008, Poland E-mail: miroslawa.puskulluoglu@uj.edu.pl

Key words: cetuximab, colorectal cancer, hypomagnesemia, epidermal growth factor receptor, metastases

\section{Introduction}

Epidermal growth factor receptor (EGFR) is a transmembrane glycoprotein, with an intracellular component that acts as a tyrosine kinase (1). As the EGFR/K-ras pathway is commonly activated in metastatic colorectal cancer (mCRC), it is an attractive target for molecular therapy (1).

$\mathrm{CRC}$ is one of the most common malignancies in men and women (2). Cetuximab, a monoclonal antibody (mAb) directed against EGFR, has shown activity in monotherapy and in combination with chemotherapy (chemoimmunotherapy) in various lines of mCRC treatment (3-6). An analysis of Cetuximab Combined with Irinotecan in First-Line Therapy for Metastatic Colorectal Cancer (CRYSTAL) phase III and Oxaliplatin and Cetuximab in First-Line Treatment of mCRC (OPUS) phase II randomized clinical trials showed statistically significant improvement in overall survival (OS), progression-free survival (PFS) and overall response rate (ORR) in patients without K-ras mutation receiving cetuximab with first-line chemotherapy (6). The CO.17 trial proved that cetuximab monotherapy administered following progression on chemotherapy lines (oxaliplatin and irinotecan with 5-fluorouracil) also improves OS, PFS and ORR (5). Inhibition of the EGFR/K-ras pathway by cetuximab is connected with numerous side-effects, such as skin toxicity, diarrhea, hypomagnesemia and other dyselectrolytemias or infusion reactions (1,7). A previous study by our group (7) analyzed skin toxicity associated with cetuximab-based therapy; acnelike rash was observed at a frequency of $80 \%$ and paronychia at $20 \%$.

Hypomagnesemia may be a result of insufficient magnesium $(\mathrm{Mg})$ supplementation in the diet, hormonal imbalance, antibiotic usage or alcoholism $(8,9)$. The National Cancer Institute Common Terminology Criteria for Adverse Events (CTCAE) version 4.0 are used to grade levels of hypomagnesemia (Table I) (10). The most common symptom of hypomagnesemia is weakness. There are also other problems, including irritability, arrhythmias or metabolic and neuromuscular disorders, which may be revealed in the case 
of higher grades of this dyselectrolytemia $(8,9)$. However, the incidence and severity of hypomagnesemia were not assessed in the aforementioned clinical trials.

Cells building the renal tubule are characterized by a high level of EGFR expression. The mechanism of $\mathrm{Mg}$ wasting during treatment with cetuximab is associated with the blockage of EGFR-dependent transient receptor potential channel 6 (TRPM6) in the nephron (Fig. 1) $(11,12)$. The blockage of this pathway results in insufficient activation of the TRPM6 epithelial ion channel and Mg wasting $(11,12)$. The process takes place mainly in the distal convoluted tube of the nephron, where the expression of TRPM6 is the greatest (Fig. 2). The other suggested mechanism is via indirect tubular nephrotoxicity (13). The fact that after cessation of therapy with cetuximab the $\mathrm{Mg}$ concentration in the plasma returns to normal suggests the reversibility of this process $(12,14)$.

The reason for the present study was to estimate the frequency and severity of hypomagnesemia among patients with mCRC treated with cetuximab. The study also aimed to measure the extent of serum $\mathrm{Mg}$ assessment in this group of patients.

\section{Patients and methods}

Patients. Between October 2009 and June 2013, a retrospective analysis of the records of 52 patients from the Department of Clinical Oncology, University Hospital, Jagiellonian University Medical College (Kraków, Poland) was performed. The inclusion criteria for the study were as follows: An age of $\geq 18$ years, a diagnosis of CRC confirmed by an available histopathological report, no $K$-ras mutation, presence of metastases on diagnostic imaging (magnetic resonance imaging, computed tomography, positron emission tomography or bone scintigraphy), and receipt of at least 2 doses of cetuximab. The exclusion criteria included: Concurrent malignancies (also in the past), malabsorption or genetic $\mathrm{Mg}$ wasting syndromes, a history of hypomagnesemia prior to the treatment, alcoholism, diarrhea (grade 3 or greater according to CTCAE v.4.0) during the 2 months prior to the start of treatment and while on the treatment with cetuximab, concurrent administration of diuretics (thiazide, loop diuretics), and a lack of consent to participate in the study.

The analyzed factors included: Sociodemographic data, localization of the primary tumor and metastases, clinical staging according to the 7th edition of Tumor-Node-Metastasis system (15), type of treatment received (including line of systemic treatment), reason for therapy ending, the presence of hypomagnesemia associated with cetuximab therapy and its intensity. Hypomagnesemia was classified according to the CTCAE v.4.0 (Table I) (10).

Statistical analysis. Statistical evaluation was conducted using computer software Statistica 11.0 PL (StatSoft Poland, Krakow, Poland). Descriptive statistics are used in the form of percentage distribution, range or mean \pm standard deviation. When comparing the quantitative variables, Student's t-test was applied; if there was absence of a normal distribution of factors, the Mann-Whitney U test was used. To check the association between quantitative variables, Spearman' test was conducted and the $\chi^{2}$ test was applied when comparing

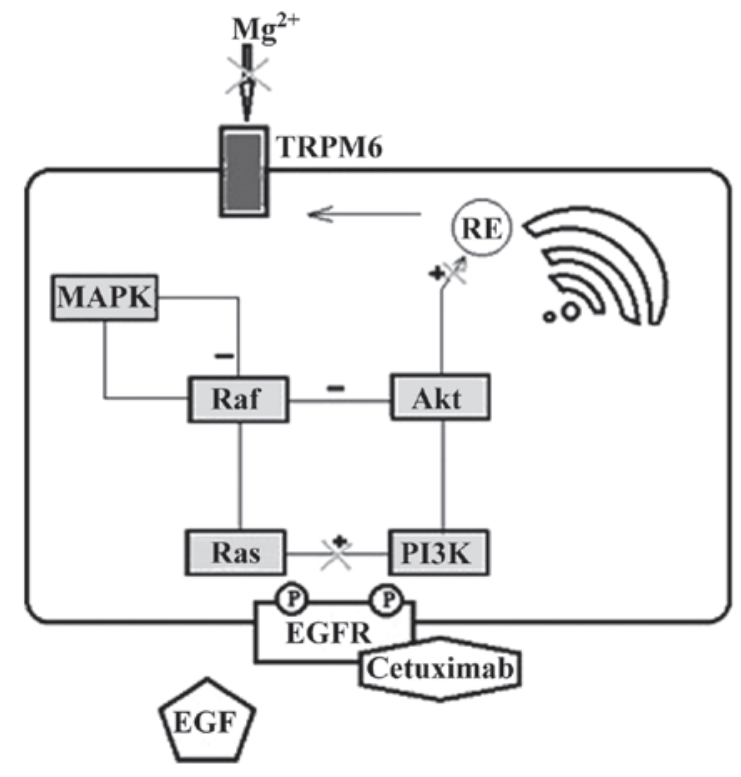

Figure 1. Mechanism of magnesium $(\mathrm{Mg})$ wasting during treatment with cetuximab, associated with the blockage of epidermal growth factor receptor (EGFR)-dependent transient receptor potential channel 6 (TRPM6). Cetuximab blocks EGFR. As a consequence, Ras and mitogen-activated protein kinase (MAPK) signaling pathways are not active and cannot affect TRPM6, which is responsible for magnesium reabsorption in the apical membranes of the cells of the distal convoluted tube. P, phosphorylated tyrosine kinase; PI3K, phosphoinositide 3-kinase; RE, endoplasmatic reticulum.

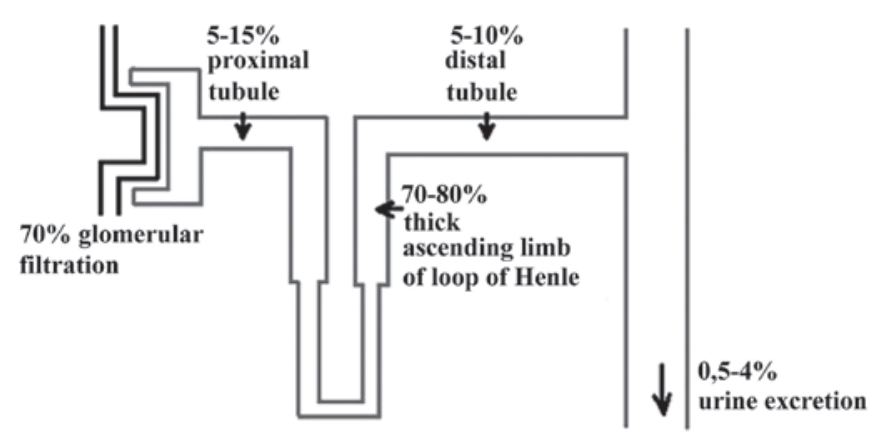

Figure 2. Magnesium reabsorption in the nephron.

qualitative variables. Factors potentially associated with the risk of developing hypomagnesemia were also assessed using logistic regression analysis. $\mathrm{P}<0.05$ was used to indicate a statistically significant difference.

Ethical approval. The present study was approved by the Jagiellonian University Medical College Ethical Committee (registry number, KB/254/B/2011). The data was collected and analyzed in accordance with the ethical standards laid down in the 1964 Declaration of Helsinki with its amendments.

Literature search. A literature search of the MEDLINE database (between January 2005 and May 2014; http://www. ncbi.nlm.nih.gov/pubmedhealth/; accessed 1st June 2014) and UpToDate (http://www.uptodate.com/; accessed 1st June 2014) was performed to find an association between treatment with cetuximab and hypomagnesemia. The key words 'anti-EGFR', 'cetuximab', 'hypomagnesemia', 'magnesium', 'metastases', 
Table I. Grades of hypomagnesemia according to common terminology criteria for adverse events v.4.0.

\begin{tabular}{lc}
\hline Grade & Hypomagnesemia \\
\hline 1 & $<\mathrm{LLN}-1.2 \mathrm{mg} / \mathrm{dl},<\mathrm{LLN}-0.5 \mathrm{mmol} / 1$ \\
2 & $<1.2-0.9 \mathrm{mg} / \mathrm{dl},<0.5-0.4 \mathrm{mmol} / 1$ \\
3 & $<0.9-0.7 \mathrm{mg} / \mathrm{dl},<0.4-0.3 \mathrm{mmol} / 1$ \\
4 & $<0.7 \mathrm{mg} / \mathrm{dl},<0.3 \mathrm{mmol} / 1$, \\
5 & life-threatening consequences \\
& Mortality \\
\hline
\end{tabular}

LLN, lower limit of normal.

'colorectal cancer', 'colon cancer', 'monoclonal antibody' and 'TRPM6' were used in various combinations.

\section{Results}

Of the 52 patients analyzed, 27 patients who fulfilled all the inclusion criteria and none of the exclusion criteria were enrolled into the study. In the excluded cases, 21 lacked a serum $\mathrm{Mg}$ level assessment, and the remainder developed grade 3 diarrhea or an anaphylactic reaction.

Table II shows the baseline characteristics of the study population, which was composed of 7 females and 20 males, with a median ( \pm standard deviation) age of $55.0 \pm 11.6$ years. Cetuximab was administered as a palliative regiment at a standard dose of $400 \mathrm{mg} / \mathrm{m}^{2}$ as a first dose and at $250 \mathrm{mg} / \mathrm{m}^{2}$ in each subsequent dose regardless of whether it was administered as monotherapy or in combination with standard chemotherapy. Chemotherapy regimens were based on irinotecan, oxaliplatin or capecytabine alone. The main reason for treatment termination in the patients treated with monotherapy or immunochemotherapy was progression of the disease (92.6\%). The median duration of treatment with cetuximab was 98 days (range, 15-546).

In $29.6 \%$ of all patients randomly assessed (more than once), the $\mathrm{Mg}$ level indicated hypomagnesemia. The majority of cases (22.2\%) were grade 1 according to CTCAE v.4.0, while 1 patient of grade 2 and 1 patient of grade 3 was revealed.

There was no statistically significant correlation between the presence of hypomagnesemia (none vs. any) or the grade of hypomagnesemia and patient age ( $\geq 55$ vs. $<55$ years; $P=0.1$ and $\mathrm{P}=0.1$, respectively), duration of treatment $(\mathrm{P}=0.9$ and $\mathrm{P}=0.3$, respectively), type of treatment (monotherapy vs. in combination with chemotherapy; $\mathrm{P}=0.3$ and $\mathrm{P}=0.6$, respectively), line of systemic treatment $(\mathrm{P}=0.3$ and $\mathrm{P}=0.2$, respectively), localization of primary tumor (rectum vs. colon; $\mathrm{P}=0.6$ and $\mathrm{P}=0.6$, respectively) or metastases (liver vs. other localizations; $\mathrm{P}=0.3$ and $\mathrm{P}=0.3$, respectively), and number of metastases ( 1 vs. $>1$; $\mathrm{P}=0.6$ and $\mathrm{P}=0.9$, respectively). There was an upward trend in a logistic regression model showing that the risk of developing hypomagnesemia increases with age (odds ratio, 1.10; $95 \%$ confidence interval, 0.97-1.25). However, the trend did not reach statistical significance $(\mathrm{P}=0.1)$.

None of the patients had the treatment discontinued due to the hypomagnesemia.
Table II. Baseline characteristics of the studied population.

\begin{tabular}{|c|c|}
\hline Parameter & Value \\
\hline \multicolumn{2}{|l|}{ Age, years } \\
\hline Median & 55.0 \\
\hline Range & $27-72$ \\
\hline \multicolumn{2}{|l|}{ Gender, n (\%) } \\
\hline Women & $7(25.9)$ \\
\hline Men & $20(74.1)$ \\
\hline \multicolumn{2}{|c|}{ Primary tumor localization, $\mathrm{n}(\%)$} \\
\hline Rectum & $13(48.1)$ \\
\hline Colon & $14(51.9)$ \\
\hline \multicolumn{2}{|c|}{ No. of organs involved with metastases, n (\%) } \\
\hline 1 & $21(77.8)$ \\
\hline$>1$ & $6(22.2)$ \\
\hline \multicolumn{2}{|c|}{ Location of metastases ${ }^{\mathrm{a}}, \mathrm{n}(\%)$} \\
\hline Liver & $18(66.7)$ \\
\hline Other & $14(51.9)$ \\
\hline \multicolumn{2}{|c|}{ Cetuximab treatment line, n (\%) } \\
\hline 1 & $9(33.3)$ \\
\hline$>1$ & $18(66.7)$ \\
\hline \multicolumn{2}{|l|}{ Type of therapy, n (\%) } \\
\hline Monotherapy & $4(14.8)$ \\
\hline Chemoimmunotherapy & $23(85.2)$ \\
\hline \multicolumn{2}{|c|}{ Reason for treatment ending, $\mathrm{n}(\%)$} \\
\hline Cancer progression & $25(92.6)$ \\
\hline Side-effects & $0(0.0)$ \\
\hline Decision of a physician & $1(3.7)$ \\
\hline Lack of data & $1(3.7)$ \\
\hline
\end{tabular}

an 11 cases patients had more than one site of metastatic disease.

\section{Discussion}

Targeted therapy with mAbs has become a widely used treatment option for cancer patients. In comparison with standard chemotherapy, targeted drugs show lower risk of severe systemic adverse effects. First suggestions with regard to the requirement for $\mathrm{Mg}$ measurement and supplementation appeared in 2005 (16). The summary of product characteristics produced for Erbitux (cetuximab) estimates the frequency of hypomagnesemia in $>10 \%$ of patients treated with the drug (17). Table III (17-29) shows the results of other studies regarding hypomagnesemia as a side-effect of cetuximab. In the available results of retrospective studies, the percentage of patients with any grade of hypomagnesemia varied from $6.3-93.3 \%$ with grade $3 / 4$ in 0 to $27 \%(16,25-30)$. One of the first studies of this dyselectrolytemia associated with cetuximab by Fakih et al (14) showed a high incidence of grade 3 and 4 compared with later studies (27\%). This may be due to the fact that only patients with both baseline $\mathrm{Mg}$ level and level assessed during the treatment were included. As checking the serum $\mathrm{Mg}$ concentration was not mandatory at the time of this study, it may be hypothesized that patients with baseline 


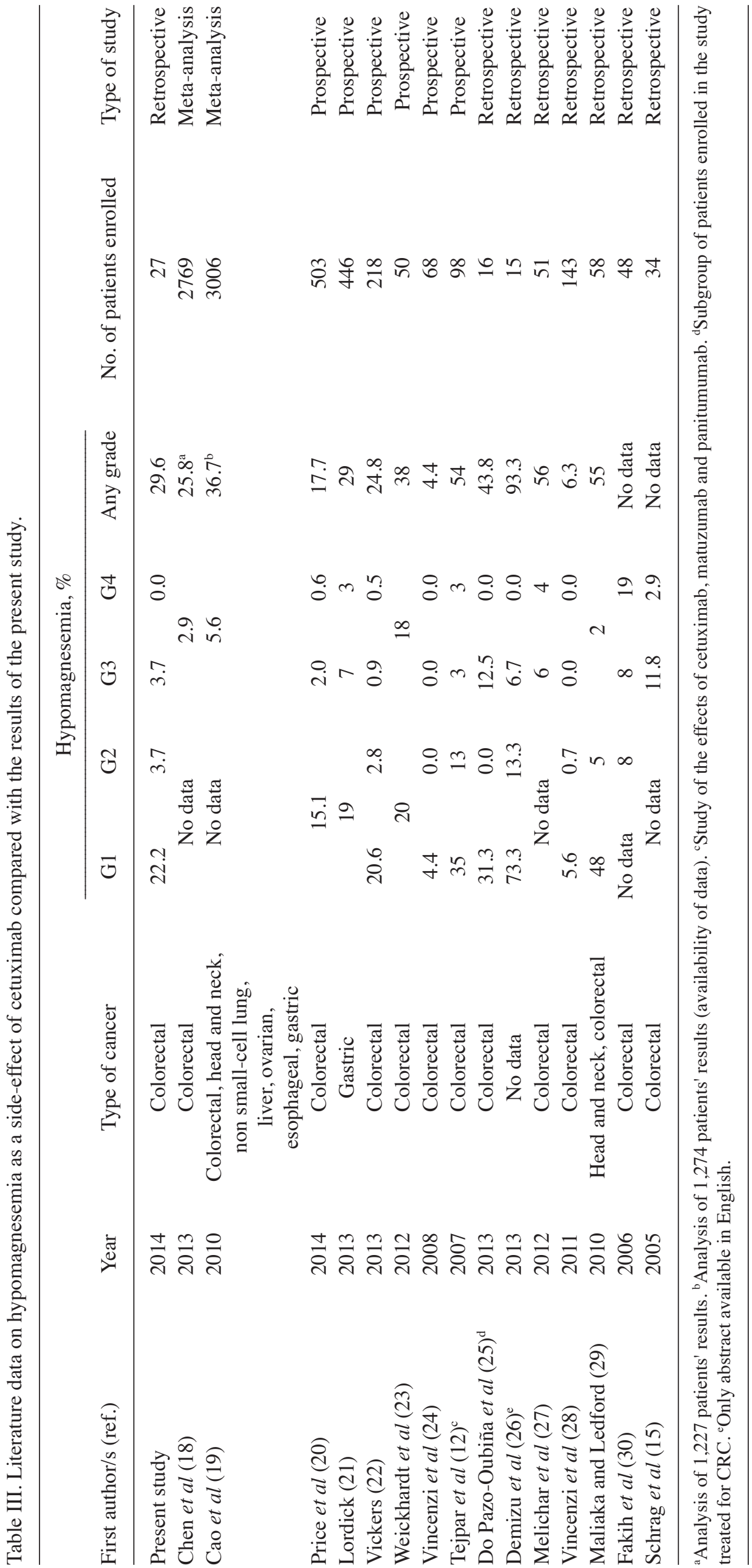


levels were those more prone to suffer from hypomagnesemia due to other causes or had dyselectrolytemia found in past laboratory tests. The same inclusion criteria were introduced in a study by do Pazo-Oubiña et al, however, in this study the incidence was higher for grade 1 and 3 , with no patient suffering from grade 4 (25). To omit this potential bias, in the present study, the enrollment of patients without baseline $\mathrm{Mg}$ level (but without hypomagnesemia in the history) was also decided upon. Taking into consideration only a sub-group with baseline assessment, the incidence in the present study was slightly higher, but did not reach the levels shown in the two aforementioned studies (data not shown).

Notably, prospective studies also showed significant discrepancies in the assessment of hypomagnesemia incidence, with any grade found in 4.4 to $54 \%$ of patients $(12,20-24)$. Even when not taking into consideration the study by Tejpar et al, which checked the effects of cetuximab, matuzumab and panitumumab, the differences are significant (4.4 to $38 \%$ ). The present data are the closest to those obtained by Vickers et al (22). Building on their research and conclusions, the huge differences in results [for example when compared with the studies by Vincenzi et al $(24,28)]$ may be associated with different baseline serum $\mathrm{Mg}$ concentrations or applied types of concomitant and past systemic treatments.

Vickers et al (22) performed a sub-group analysis, which found hypomagnesemia to be more commonly presented in patients without K-ras mutation (19 vs. 27\%; the difference was not statistically assessed for the significance).

Looking at the two meta-analyses found in the literature search, any grade of hypomagnesemia was observed in 25.8 and $36.7 \%$ of patients, and grade $3 / 4$ in 2.9 and $5.6 \%$ of patients, respectively $(18,19)$. These results are consistent with the present study observations. Grades 1 and 2 were estimated in none of these meta-analyses $(18,19)$. According to Chen et al (18), patients with mCRC have a higher incidence of hypomagnesemia grade $3 / 4$ than patients with other malignancies. Comparing their results obtained for patients with mCRC with an earlier meta-analysis performed by Cao et al (19) on a group of patients with various malignancies it can be noted that this incidence was actually higher in the latter study. Additionally, a retrospective study by Maliakal and Ledford that also enrolled patients with head and neck cancer had one of the highest percentages of patients with hypomagnesemia. Table III presents only a sub-group of patients from the study by do Pazo-Oubiña et al (mCRC treated with cetuximab) (25). In this study, patients with head and neck carcinoma were also enrolled, and it was concluded that overall hypomagnesemia was less common in $\mathrm{mCRC}$ patients than head and neck cancer patients (43.8 vs. $72.2 \%)$.

In the present study, no grade 4 hypomagnesemia was observed, which is generally consistent with the majority of other studies where this metabolic complication was a rare event at grade 4 . Only one study estimated the level of grade $3 / 4$ hypomagnesemia at $27 \%$ (30). Thus, it may be assumed that $\mathrm{Mg}$ depletion is a common, but not life-threatening complication in the population of patients treated with EGFR-targeting mAbs. Also, certain other studies indicated that there was no requirement for therapy termination (or reduction) due to hypomagnesemia caused by cetuximab (12). However, it has also been indicated that this metabolic side-effect may influence treatment in severely affected individuals (12).

The main reason for $\mathrm{Mg}$ wasting is the blockage of EGFR-dependent TRPM6 in the nephron resulting in impaired renal reabsorption $(12,22)$. There are also suggestions that blocking EGFR by cetuximab may affect the absorption of $\mathrm{Mg}$ in the gut $(12,16)$, or that the tubular damage in the kidneys is caused by mAb precipitation (14). Few factors that may predispose to the development and severity of hypomagnesemia during the treatment with cetuximab are taken into consideration. Certain studies propose that concurrent chemotherapy with platinum agents is indicated, as these affect $\mathrm{Mg}$ level most significantly (31). Also, the time factor appears to play an important role $(30,31)$. Results of one study showed an increase in hypomagnesemia incidence proportional to the duration of the treatment (30). No such association was observed in the present study.

Tejpar et al found an association between an older patient age and Mg wasting (12). This trend was also observed in the present study, although it was not statistically significant. The connection appears to be logical, as ageing is also connected with other conditions leading to $\mathrm{Mg}$ loss, such as glomerulosclerosis or deterioration in renal function (32). Notably, higher baseline level may be connected with more prompt $\mathrm{Mg}$ reduction $(12,22)$.

Hypomagnesemia resulting from EGFR blockage may be a class effect for all $\mathrm{mAbs}$ directed against this receptor. Exact differences between mAbs have not yet been assessed (12).

There are no reliable and precise recommendations concerning $\mathrm{Mg}$ measurement and supplementation in patients with $\mathrm{mCRC}$ receiving anti-EGFR $\mathrm{mAbs}$. The Erbitux summary of product characteristics claims only that the assessment of serum $\mathrm{Mg}$ level (and that of other electrolytes) prior to and periodically during the treatment with cetuximab and, as appropriate, supplementation of electrolytes is recommended (17). Additionally, studies have made suggestions that regular Mg screening should be performed, particularly in patients treated simultaneously with platinum-based agents $(25,31)$. The suggested interval for serum $\mathrm{Mg}$ measurement is 4-8 weeks plus the baseline level (25).

As there are no particular recommendations for $\mathrm{Mg}$ replacement in this particular group of patients, it appears reasonable to follow general guidelines for $\mathrm{Mg}$ supplementation (9). Certain cancer centers have created their own treatment guidelines (26,30). Fakih et al (30) administered intravenous $\mathrm{Mg}$ sulfate daily or 3 times/week, at 6-10 g per dose, in patients with grade 3 and 4 hypomagnesemia. Also Tejpar et al (12) performed daily intravenous $\mathrm{Mg}$ supplementation in severely affected individuals. It is notable that oral $\mathrm{Mg}$ supplementation in cancer patients may be ineffective due to diarrhea or malabsorption $(12,30)$. Results of the study comparing oral low- and high-dose $\mathrm{Mg}$ supplementation in this group of patients are expected to be published (12).

Due to its retrospective character and small population size, the present study is characterized by certain limitations. Data regarding sociodemographic status, as well as information on the treatment and disease were gathered from medical records. Sporadically, the information was incomplete. Only half the patients $(27 / 52 ; 51.9 \%)$ entered the study. The reason 
for this was mainly as there was no information on $\mathrm{Mg}$ level due to the lack of recommendations suggesting regular $\mathrm{Mg}$ measurement. However, previously described studies on cetuximab efficacy have also not carefully assessed the frequency of hypomagnesemia (3-6). In a study performed by Schrag et al, only $22.1 \%$ of patients (34 patients) entered the retrospective studies assessing $\mathrm{Mg}$ level $(16,25)$, while in a recently published study by do Pazo-Oubiña et al, $33.8 \%$ of patients (68 patients) received $\mathrm{mAb}$ anti-EGFR. Currently, all patients in the Department of Clinical Oncology, University Hospital of Krakow, undergo regular Mg level assessment once every 4 weeks, and in the case of any abnormalities, every 2 weeks (prior to every cetuximab infusion), or more often if required.

It would also be interesting to observe the frequency and intensity of $\mathrm{Mg}$ decrease from its baseline level prior to the treatment; however, these data were not present in all patient records. One study on 98 patients with mCRC revealed a decrease in serum $\mathrm{Mg}$ concentration in $97 \%$ of patients during the treatment with mAbs directed against EGFR (12).

Published data on the effect of $\mathrm{Mg}$ level on tumor growth are inconclusive (33). There are studies claiming that early hypomagnesemia may work as an inexpensive positive predictive factor for the treatment with cetuximab $(24,28)$. This connection has already been described for the skin-related toxicity caused by this mAb (7). One study has suggested that hypomagnesemia may function in decreasing the proliferation of cells (33). However, other recently published studies suggest an opposite association and a decrease in OS time in patients with hypomagnesemia (22). Vickers et al (22) hypothesized that the predictive meaning of hypomagnesemia may be associated with the severity of this side-effect, with lower levels being associated with better treatment outcome and higher levels with worse treatment outcome. However, due to the limited number of patients and a variety of treatment lines, it was not possible to perform statistical analysis of the possible correlations between protocol type/response to the treatment and hypomagnesemia occurrence or grading.

Finally, it should be noted that the prevalence of hypomagnesemia in the healthy population has been estimated as between 2.5 and $15 \%$. A review by Saif suggested an even higher prevalence among cancer patients due to higher urinary and gastrointestinal loss (e.g., diarrhea), malnutrition and poor dietary intake (9). Patients with neoplastic diseases also commonly present with weakness/fatigue, which is the most common symptom of mild hypomagnesemia. It would be extremely difficult to estimate the frequency of this side-effect of cetuximab in a retrospective study, and of other symptoms, such as irritability. For this reason, similar to certain other studies (12), it was decided against collecting data on hypomagnesemia symptoms in the present study.

The results shown in this study and previously published records regarding skin-related toxicity (7) demonstrate that cetuximab-related side-effects present their specific characteristics regardless of whether the drug is used as a monotherapy or in combination with standard chemotherapy. It is essential to know the main symptoms of hypomagnesemia in order to ensure the safety of treatment with cetuximab. Physicians should focus on actively searching for hypomagnesemia and other typical adverse effects in this group of patients. The data regarding this side-effect remain limited, with the hypomagnesemia incidence being assessed at between 4.4 to $93.3 \%$, depending on the study. These metabolic complications are not usually life-threatening, nor do they lead to treatment termination, but require monitoring and treatment. As the extent of $\mathrm{Mg}$ monitoring in patients treated with mAbs directed against EGFR is insufficient, it is reasonable to introduce recommendations concerning $\mathrm{Mg}$ measurement and supplementation in this population. Physicians should remember that hypomagnesemia may be revealed as a side-effect of cancer treatment, not only as a result of diarrhea or malabsorption.

\section{Acknowledgements}

The authors would like to thank Dr Agnieszka Pacek and Dr Magdalena Kozioł for their invaluable support in data collection, and Ms. Joanna Gołąb for editing the original manuscript.

\section{References}

1. Fakih $\mathrm{M}$ and Vincent $\mathrm{M}$ : Adverse events associated with anti-EGFR therapies for the treatment of metastatic colorectal cancer. Curr Oncol 17 (Suppl 1): S18-S30, 2010.

2. Boyle P and Langman JS: ABC of colorectal cancer: Epidemiology. BMJ 321: 805-808, 2000.

3. Saltz LB, Meropol NJ, Loehrer PJ Sr, Needle MN, Kopit J and Mayer RJ: Phase II trial of cetuximab in patients with refractory colorectal cancer that expresses the epidermal growth factor receptor. J Clin Oncol 22: 1201-1208, 2004.

4. Cunningham D, Humblet Y, Siena S, Khayat D, Bleiberg H, Santoro A, Bets D, Mueser M, Harstrick A, Verslype C, et al: Cetuximab monotherapy and cetuximab plus irinotecan in irinotecan-refractory metastatic colorectal cancer. N Engl J Med 351: 337-345, 2004.

5. Jonker DJ, O'Callaghan CJ, Karapetis CS, Zalcberg JR, Tu D, Au HJ, Berry SR, Krahn M, Price T, Simes RJ, et al: Cetuximab for the treatment of colorectal cancer. N Engl J Med 357: 2040-2048, 2007.

6. Bokemeyer C, Van Cutsem E, Rougier P, Ciardiello F, Heeger S, Schlichting M, Celik I and Köhne CH: Addition of cetuximab to chemotherapy as first-line treatment for KRAS wild-type metastatic colorectal cancer: Pooled analysis of the CRYSTAL and OPUS randomized clinical trials. Eur J Cancer 48: 1466-1475, 2012.

7. Pacek A, Kozioł M, Püsküllüoğlu M, Tomaszewski KA Ochenduszko S, Zygulska AL and Krzemieniecki K: Assessment of skin-related toxicity in patients with metastatic colorectal cancer treated with cetuximab. Acta Dermatovenerol Croat 22: 137-144, 2014.

8. Martin KJ, González EA and Slatopolsky E: Clinical consequences and management of hypomagnesemia. J Am Soc Nephrol 20: 2291-2295, 2009.

9. Saif MW: Management of hypomagnesemia in cancer patients receiving chemotherapy. J Support Oncol 6: 243-248, 2008.

10. National Cancer Institute Common Toxicity Criteria Version 4.0; May 28, 2009. http://ctep.cancer.gov/protocolDevelopment/electronic_applications/ctc.htm. Accessed May 2, 2014

11. Groenestege WM, Thébault S, van der Wijst J, van den Berg D, Janssen R, Tejpar S, van den Heuvel LP, van Cutsem E, Hoenderop JG, Knoers NV, et al: Impaired basolateral sorting of pro-EGF causes isolated recessive renal hypomagnesemia. J Clin Invest 117: 2260-2267, 2007.

12. Tejpar S, Piessevaux H, Claes K, Piront P, Hoenderop JG, Verslype $\mathrm{C}$ and Van Cutsem E: Magnesium wasting associated with epidermal-growth-factor receptor-targeting antibodies in colorectal cancer: A prospective study. Lancet Oncol 8: 387-394, 2007.

13. Yang XD, Jia C, Corvalan JR, Wang P and Davis CG: Development of ABX-EGF, a fully human anti-EGF receptor monoclonal antibody, for cancer therapy. Crit Rev Oncol Hematol 38: 17-23, 2001.

14. Fakih M: Anty-EGFR monoclonal antibody-induced hypomagnesaemia. Lancet Oncol 8: 366-367, 2007. 
15. Edge S, Byrd DR, Compton CC, Fritz AG, Greene FL and Trotti A: AJCC Cancer Staging Manual. 7th edition. Springer, New York, NY, p143, 2010.

16. Schrag D, Chung KY, Flombaum C and Saltz L: Cetuximab therapy and symptomatic hypomagnesemia. J Natl Cancer Inst 97: 1221-1224, 2005.

17. Erbitux - Summary of product characteristics. http://www. ema.europa.eu/docs/en_GB/document_library/EPAR_-_Product Information/human/000558/WC500029119.pdf. Accessed May 2, 2014

18. Chen P, Wang L, Li H, Liu B and Zou Z: Incidence and risk of hypomagnesemia in advanced cancer patients treated with cetuximab: A meta-analysis. Oncol Lett 5: 1915-1920, 2013.

19. Cao Y, Liao C, Tan A, Liu L and Gao F: Meta-analysis of incidence and risk of hypomagnesemia with cetuximab for advanced cancer. Chemotherapy 56: 459-465, 2010.

20. Price TJ, Peeters M, Kim TW, Li J, Cascinu S, Ruff P, Suresh AS, Thomas A, Tjulandin S, Zhang K, et al: Panitumumab versus cetuximab in patients with chemotherapy-refractory wild-type KRAS exon 2 metastatic colorectal cancer (ASPECCT): A randomised, multicentre, open-label, non-inferiority phase 3 study. Lancet Oncol 15: 569-579, 2014.

21. Lordick F, Kang YK, Chung HC, Salman P, Oh SC, Bodoky G, Kurteva G, Volovat C, Moiseyenko VM, Gorbunova V, et al Capecitabine and cisplatin with or without cetuximab for patients with previously untreated advanced gastric cancer (EXPAND): A randomised, open-label phase 3 trial. Lancet Oncol 14 490-499, 2013

22. Vickers MM, Karapetis CS, Tu D, O'Callaghan CJ, Price TJ, Tebbutt NC, Van Hazel G, Shapiro JD, Pavlakis N, Gibbs P, et al: Association of hypomagnesemia with inferior survival in a phase III, randomized study of cetuximab plus best supportive care versus best supportive care alone: NCIC CTG/AGITG CO.17. Ann Oncol 24: 953-960, 2013.

23. Weickhardt AJ, Price TJ, Chong G, Gebski V, Pavlakis N, Johns TG, Azad A, Skrinos E, Fluck K, Dobrovic A, et al: Dual targeting of the epidermal growth factor receptor using the combination of cetuximab and erlotinib: Preclinical evaluation and results of the phase II DUX study in chemotherapy-refractory, advanced colorectal cancer. J Clin Oncol 30: $1505-1512,2012$.
24. Vincenzi B, Santini D, Galluzzo S, Russo A, Fulfaro F, Silletta M, Battistoni F, Rocci L, Zobel BB, Adamo V, et al: Early magnesium reduction in advanced colorectal cancer patients treated with cetuximab plus irinotecan as predictive factor of efficacy and outcome. Clin Cancer Res 14: 4219-4224, 2008.

25. Do Pazo-Oubiña F, Estefanell-Tejero A, Riu-Viladoms G, Anglada-Martínez H, Molas-Ferrer G and Creus-Baró N: Magnesium monitoring practice in monoclonal anti-epidermal growth factor receptor antibodies therapy. J Clin Pharm Ther 38: 101-103, 2013.

26. Demizu M, Ueda $H$, Osawa $M$, Chihara S, Igarashi $T$, Yano K, Kimura F, Tanaka N and Hiratsuka M: Effect of magnesium supplementation on early-stage hypomagnesemia in patients treated with cetuximab. Gan To Kagaku Ryoho 40: 897-900, 2013

27. Melichar B, Králíčková P, Hyšpler R, Kalábová H, Cerman J Jr, Holečková $\mathrm{P}$, Studentová $\mathrm{H}$ and Malírová E: Hypomagnesaemia in patients with metastatic colorectal carcinoma treated with cetuximab. Hepatogastroenterology 59: 366-371, 2012.

28. Vincenzi B, Galluzzo S, Santini D, Rocci L, Loupakis F Correale P, Addeo R, Zoccoli A, Napolitano A, Graziano F, et al: Early magnesium modifications as a surrogate marker of efficacy of cetuximab-based anticancer treatment in KRAS wild-type advanced colorectal cancer patients. Ann Oncol 22: 1141-1146, 2011.

29. Maliakal P and Ledford A: Electrolyte and protein imbalance following anti-EGFR therapy in cancer patients: A comparative study. Exp Ther Med 1: 307-311, 2010.

30. Fakih MG, Wilding G and Lombardo J: Cetuximab-induced hypomagnesemia in patients with colorectal cancer. Clin Colorectal Cancer 6: 152-156, 2006.

31. Stintzing S, Fischhaber D, Mook C, Modest DP, Giessen C, Schulz C, Haas M, Boeck S, Michl M, Stemmler J, et al: Clinical relevance and utility of cetuximab-related changes in magnesium and calcium serum levels. Anticancer Drugs 24: 969-974, 2013.

32. Thomas SE, Anderson S, Gordon KL, Oyama TT, Shankland SJ and Johnson RJ: Tubulointerstitial disease in aging: Evidence for underlying peritubular capillary damage, a potential role for renal ischemia. J Am Soc Nephrol 9: 231-242, 1998.

33. Wolf FI, Cittadini AR and Maier JA: Magnesium and tumors: Ally or foe? Cancer Treat Rev 35: 378-382, 2009. 